\title{
Computation of Partial Equilibrium Solidification with Complete Interstitial and Negligible Substitutional Solute Back Diffusion
}

\author{
Qing Chen and Bo Sundman
}

Department of Materials Science and Engineering, Royal Institute of Technology, S-100 44, Stockholm, Sweden

A simple numerical scheme is presented to simulate partial equilibrium solidification with complete interstitial and negligible substitutional solute back diffusion in multi-component and multi-phase systems. Based on this scheme, a computing tool capable of using Thermo-Calc databases directly has been developed for the estimation of solidification behavior of steels and other interstitial-containing alloys. Agreements between calculated and experimental as well as DICTRA results have been obtained on the microsegregation, fraction of eutectic, and freezing range of several steels. This suggests that the partial equilibrium assumption and proposed numerical scheme are reasonable and satisfactory, and confirms that the carbon back diffusion plays a very important role in the solidification of steels.

(Received December 12, 2001; Accepted January 25, 2002)

Keywords: numerical simulation, solidification, thermodynamics, microsegregation, steel

\section{Introduction}

The exact behavior of a solidifying system under local equilibrium conditions is controlled by the thermodynamical properties and solute diffusion in both liquid and solid phases and can only be predicted by using software with full integration of thermodynamics and kinetics, such as DICTRA. ${ }^{1)}$ However, in practice the solidification process can often be approximated in simple and efficient ways without evoking time-consuming kinetic calculations. The two extremes are equilibrium (lever-rule) and non-equilibrium (Scheil-Gulliver) methods. ${ }^{2-4)}$ They both assume uniform solute distribution in the liquid; and this is usually reasonable due to the large atomic mobility and presence of convection in the liquid. For the solid, the lever-rule method assumes that the solute diffusion is also so rapid that uniform composition is maintained, which is reasonable for interstitial solutes like $\mathrm{C}$ and $\mathrm{N}$ only. For substitutional solutes in the solid, on the other hand, diffusion is much slower and can often be neglected. In this case, each infinitely small portion of the solid, once formed, is expected to retain the same composition through the solidification course. This is the other basic assumption of the Scheil-Gulliver method.

The Scheil-Gulliver simulation with variable partition coefficients calculated from thermodynamic databases often gives a reasonable approximation to the normal solidification behavior in $\mathrm{Al}$ and Ni-based alloys, ${ }^{5)}$ but is by no means successful in the investigation of steels where the back diffusion of interstitial carbon must be taken into account. ${ }^{6)}$ A natural and more realistic approximation to the situation in steels is expected to be a partial equilibrium one, i.e. complete interstitial but negligible substitutional solute back diffusion in solids. This approach has been applied in a crude way by Fredriksson and Hellner ${ }^{7)}$ to study the influence of carbon on the segregation of chromium in steels through combining Scheil-Gulliver's segregation equation for $\mathrm{Cr}$ and the lever rule for $\mathrm{C}$ together with some phase diagram information. In order to realize the partial equilibrium approach exactly, a numerical scheme with the aid of a thermodynamic database must be adopted because it is the chemical potential or ac- tivity rather than composition of the interstitial that remains uniform throughout the solid. In a recent report, an effort in this direction has been described briefly by Kozeschnik. ${ }^{8)}$ The present work intends to develop a practical numerical scheme and build a computing tool for partial equilibrium solidification simulation. Application examples will be given to show that this approach is an excellent approximation to the solidification behavior of steels except for those dominated by the ferritic primary phase, where the back diffusion of substitutional solute is hardly negligible.

\section{Methodology}

There is no shortage of analytical solutions to the problem of solidification with solute back diffusion, but it seems that none of them is suitable for our purpose of partial equilibrium simulation. In this section, we will first examine the limitations of the existing analytical models and explain specifically why they should fail upon the partial equilibrium condition. A numerical method will then be introduced for Scheil-Gulliver solidification and further modified for partial equilibrium solidification.

\subsection{Limitations of analytical models}

Various analytical equations have been proposed to take into account the solute back diffusion in the solidification process over past years. They were usually derived from a binary solidification case involving only one solid phase and, consequently, can only be utilized to analyze the primary-phase solidification stage. For multi-solid-phase solidification stages, which is normally unavoidable in commercial alloys, no analytical solution is feasible so far. Even within the ability of the analytical methods, there is a pitfall with all of them if the situation of partial equilibrium is encountered in multicomponent systems.

The first of those analytical equations was developed by Brody and Flemings: ${ }^{9)}$

$$
x_{i}^{* S} / K_{i}=x_{i}^{L}=x_{i}^{0}\left\{1-\left(1-2 \alpha K_{i}\right) f_{S}\right\}^{\left(K_{i}-1\right)\left(1-2 \alpha K_{i}\right)},
$$

where $x_{i}^{* S}$ is the concentration of component $i$ in the solid 
at the liquid/solid interface, $K_{i}$ the partition coefficient, $x_{i}^{L}$ the concentration in the liquid, $x_{i}^{0}$ the initial or overall concentration, and $f_{S}$ the volume fraction of solid formed. The back-diffusion parameter $\alpha$ is a constant related to the solid diffusivity $D_{i}^{S}$ of solute element $i$, secondary dendrite arm spacing $\lambda$ and local freezing time $t_{f}$ by the expression

$$
\alpha=4 D_{i}^{S} t_{f} / \lambda^{2} \text {. }
$$

Apparently, eq. (1) cannot be applied to describe the behavior of infinitely fast-moving elements. As Clyne and Kurz ${ }^{10)}$ pointed out later, the use of eq. (1) should be limited to very slow-moving elements, otherwise solute atoms are not conserved. Clyne and Kurz examined this limitation and proposed a modified form of the Brody-Flemings equation and applied it also to fast-moving elements. They suggested that instead of using the parameter $\alpha$ inside eq. (1) one could use a function of it

$$
\Omega(\alpha)=\alpha\{1-\exp (-1 / \alpha)\}-(1 / 2) \exp (-1 / 2 \alpha) .
$$

Other simpler or more complex equations have been proposed by Ohnaka, ${ }^{11)}$ Kobayashi, ${ }^{12)}$ and Nastac and Stefanescu. ${ }^{13)}$ Asymptotical analyses of all these modified Brody-Flemings equations ${ }^{10,11)}$ or completely new ones, ${ }^{12,13)}$ lead successfully to the equilibrium or lever-rule equation if $D_{i}^{S} \rightarrow \infty$ :

$$
x_{i}^{S} / K_{i}=x_{i}^{L}=x_{i}^{0}\left(1-\left(1-K_{i}\right) f_{S}\right)^{-1},
$$

where $x_{i}^{S}$ is the concentration in the solid, and the ScheilGulliver's equation if $D_{i}^{S} \rightarrow 0$ :

$$
x_{i}^{* S} / K_{i}=x_{i}^{L}=x_{i}^{0}\left(1-f_{\mathrm{S}}\right)^{K_{i}-1} .
$$

This is appropriate in binary systems because eq. (4) holds if there is only one solute element and its diffusivity approaches infinity, and eq. (5) holds if its diffusivity is close to zero. In multi-component systems, the diffusivities of the solutes may all be approximated as infinitely large, or all negligible, or some infinitely large and some negligible. For the first scenario, the asymptotical result eq. (4) should be met for each solute element; for the second scenario, the asymptotical result eq. (5) should hold for each solute element. For the third scenario, which has here been called partial equilibrium, application of these analytical equations ${ }^{10-13)}$ results in eq. (5) for some solute elements due to $D_{i}^{S} \rightarrow 0$ and eq. (4) for the others due to $D_{i}^{S} \rightarrow \infty$. However, that cannot be a good description if there is a thermodynamic interaction between elements of the two kinds. From a thermodynamic point of view, if a solute has infinitely large atomic mobility, equilibrium shall be attained for it everywhere in the whole system, that is to say, its chemical potential or activity is uniform. For the partial equilibrium condition, the concentration of each infinitely slow-moving element varies throughout the solid according to eq. (5) and at the same time the uniformity of chemical potential should be maintained for each infinitely fast-moving element. As a consequence, the concentration of each infinitely fast-moving element could not be uniform in the solid, and thus eq. (4) cannot hold for the element $i$ even if $D_{i}^{S} \rightarrow \infty$. The fact that the asymptotical results can not hold for all the scenarios suggests that those analytical equations, which are appropriate for binary systems, lack thermodynamical consistency if applied to partial equilibrium situation in multi-component systems. Even though one may be tempted to combine eqs. (4) and (5) directly to treat the partial equilibrium solidification, that approach is thermodynamically incorrect and may lead to very different results from that of a real partial equilibrium calculation. This will be demonstrated in the Results and Discussion section.

The solute redistribution in a solidification process is very sensitive to the value of the partition coefficients used in analytical models. The partition coefficient will seldom be a constant in the temperature-composition space as assumed in all analytical models. With the advance of CALPHAD (CALculation of PHAse Diagrams) technique, ${ }^{5,14-16)}$ a number of software and thermodynamic database systems, such as Thermo-Calc, ${ }^{17)}$ are now available for the calculation of the phase equilibrium of an alloy system from which the partition coefficients can be calculated readily at any temperature and composition. Therefore, when analytical methods are employed, it is better to use them for numerical calculations by combining them with appropriate thermodynamic software and database rather than applying constant partition coefficients.

\subsection{Numerical method}

The solidification process is driven by the extraction of heat, whether the alloy is binary or multi-component, whether it involves one solid or several solids, and whether it can be approximated by conditions of equilibrium, non-equilibrium, or partial equilibrium. If we lower the temperature successively for a small degree $\Delta T$ from the liquidus temperature and calculate the phase equilibrium of the given system at each temperature step with a thermodynamic database, the equilibrium solidification path and fraction of solid as well as other interesting properties can be obtained readily. For the Scheil-Gulliver model, owing to the local equilibrium assumption at the phase interface, we can use the same technique but keep changing the composition and amount of the liquid on which a phase equilibrium calculation is performed. For the partial equilibrium model, the same strategy will be used but the change of composition and amount of the liquid at each step is different owing to the interstitial back diffusion.

\subsubsection{Scheil-Gulliver model}

Supposing we have an alloy with $m(m \geq 1)$ solute elements, an initial composition of $x_{i}^{0}(i=1, \ldots, m)$, and total amount of one mole of atoms $(N=1)$, its Scheil-Gulliver solidification process can be simulated by following the steps described below:

0 ) Locate the liquidus temperature $T_{0}$, which can be obtained together with the number of equilibrium solid phase(s), $n(n \geq 1)$, and the composition of each solid phase $S_{j}(j=1, \ldots, n),{ }^{0} x_{i}^{* S_{j}}$, by a phase equilibrium calculation on a system of initial composition and amount with the fraction of liquid set to unity. At this temperature, we have ${ }^{0} f_{S_{j}}=0,{ }^{0} f_{S}=\sum_{j}{ }^{0} f_{S_{j}}=0$, and ${ }^{0} f_{L}=1-{ }^{0} f_{S}=1$, where ${ }^{0} f_{S_{j}}$ is the amount or fraction of the solid phase $S_{j}$ (the amount will be expressed in moles and is identical to fraction), ${ }^{0} f_{S}$ the amount of solid, and ${ }^{0} f_{L}$ the amount of remaining liquid. The composition of the liquid phase, ${ }^{0} x_{i}^{L}$, is of course equal to 
$x_{i}^{0}$

1) Lower the temperature from $T_{0}$ to $T_{1}$ by a small decrement $\Delta T$ and the first small amount of solid phase $S_{j}$, $\Delta^{1} f_{S_{j}}$, is formed. The value of $\Delta^{1} f_{S_{j}}$ and the composition of the solid phase $S_{j}$ and liquid at this temperature step, ${ }^{1} x_{i}^{* S_{j}}$ and ${ }^{1} x_{i}^{L}$, can be obtained by performing phase equilibrium calculation on a system of composition ${ }^{0} x_{i}^{L}$ and amount ${ }^{0} f_{L}$. The amounts of solid formed thus far can be thought of as: ${ }^{1} f_{S_{j}}={ }^{0} f_{S_{j}}+\Delta^{1} f_{S_{j}}$ and ${ }^{1} f_{S}=\sum_{j}{ }^{1} f_{S_{j}}$. The amount of remaining liquid is ${ }^{1} f_{L}=1-{ }^{1} f_{S}$.

2) Lower the temperature from $T_{1}$ to $T_{2}$ by a small decrement $\Delta T$ and another small amount of solid phase $S_{j}$, $\Delta^{2} f_{S_{j}}$, is formed from the liquid left from step 1 , which has a composition of ${ }^{1} x_{i}^{L}$. The value of $\Delta^{2} f_{S_{j}}$ and the composition of newly formed solid phase(s) and remaining liquid, ${ }^{2} x_{i}^{* S_{j}}$ and ${ }^{2} x_{i}^{L}$, can be obtained from a phase equilibrium calculation on a system of composition ${ }^{1} x_{i}^{L}$ and amount ${ }^{1} f_{L}$. The amounts of solid formed so far are ${ }^{2} f_{S_{j}}={ }^{1} f_{S_{j}}+\Delta^{2} f_{S_{j}}$ and ${ }^{2} f_{S}=\sum_{j}{ }^{2} f_{S_{j}}$. The amount of remaining liquid is ${ }^{2} f_{L}=1-{ }^{2} f_{S}$. Since there is no diffusion in the solid, there will be no transport of atoms among newly formed portion of solid and the old ones although the chemical potential of each solute element is different in different parts of solid. The local equilibrium holds between the newly formed solid phase(s) and remaining liquid and there is no redistribution of atoms between liquid and solid phase(s).

3) Lower the temperature and repeat similar calculations until the remaining amount of liquid is less than a given threshold.

Apparently, the steps after 0) can be generalized as follows: lower the temperature from $T_{k}$ to $T_{k+1}(k \geq 0)$ by a small decrement $\Delta T$ and a small amount of solid phase $S_{j}, \Delta^{k+1} f_{S_{j}}$ will be formed from the liquid left from step $k$, which has a composition of ${ }^{k} x_{i}^{L}$. The value of $\Delta^{k+1} f_{S_{j}}$ and the composition of newly formed solid phase(s) and remaining liquid, ${ }^{k+1} x_{i}^{* S_{j}}$ and ${ }^{k+1} x_{i}^{L}$, can be obtained from a phase equilibrium calculation by setting the amount of a system to ${ }^{k} f_{L}$ and composition to ${ }^{k} x_{i}^{L}$. The amounts of solid formed thus far are ${ }^{k+1} f_{S_{j}}={ }^{k} f_{S_{j}}+\Delta^{k+1} f_{S_{j}}$ and ${ }^{k+1} f_{S}=\sum_{j}{ }^{k+1} f_{S_{j}}$. The amount of remaining liquid is ${ }^{k+1} f_{L}=1-{ }^{k+1} f_{S}$. Check if ${ }^{k+1} f_{L}$ is smaller than a given value or not. If not, continue to the next step and repeat similar calculations. Otherwise, terminate the simulation. The whole computational process is schematically drawn within the dashed line rectangle in Fig. 1.

The numerical scheme outlined above is a general one and will work in multi-component and multi-phase systems. It has been used ${ }^{18)}$ and implemented into a special module ${ }^{19)}$ inside the Thermo-Calc. The fact that this numerical scheme reduces to the Scheil-Gulliver equation for constant partition coefficients can be illustrated as follows.

From any step $k$ to $k+1$, since it is an equilibrium calculation, $\Delta^{k+1} f_{S},{ }^{k+1} x_{i}^{* S},{ }^{k+1} x_{i}^{L},{ }^{k} x_{i}^{L}$ and ${ }^{k} f_{L}$ are related to each other by the lever-rule. Here subscript $j$ of $S$ is omitted because only one solid phase is considered in the analytical Scheil-Gulliver method. As a result, we have

$$
\begin{aligned}
\left({ }^{k} x_{i}^{L}\right. & \left.-{ }^{k+1} x_{i}^{* S}\right) \Delta^{k+1} f_{S} /{ }^{k} f_{L} \\
& =\left({ }^{k+1} x_{i}^{L}-{ }^{k} x_{i}^{L}\right)\left(1-\Delta^{k+1} f_{S} /{ }^{k} f_{L}\right),
\end{aligned}
$$

which may be rearranged into

$$
\left({ }^{k+1} x_{i}^{L}-{ }^{k+1} x_{i}^{* S}\right) \Delta^{k+1} f_{S}=\left({ }^{k+1} x_{i}^{L}-{ }^{k} x_{i}^{L}\right)^{k} f_{L} .
$$

Substitution of ${ }^{k} f_{L}=1-{ }^{k} f_{S}$ and ${ }^{k+1} x_{i}^{* S}={ }^{k+1} K_{i}{ }^{k+1} x_{i}^{L}$ into eq. (7) gives

$$
\left({ }^{k+1} x_{i}^{L}-{ }^{k+1} K_{i}{ }^{k+1} x_{i}^{L}\right) \Delta^{k+1} f_{S}=\left({ }^{k+1} x_{i}^{L}-{ }^{k} x_{i}^{L}\right)\left(1-{ }^{k} f_{S}\right),
$$

which can be rewritten as

$$
{ }^{k+1} x_{i}^{L}\left(1-{ }^{k+1} K_{i}\right) \Delta^{k+1} f_{S}=\Delta^{k+1} x_{i}^{L}\left(1-{ }^{k} f_{S}\right) .
$$

If the step is small enough, the step superscript can be ignored and we get

$$
\left(1-K_{i}\right) \frac{d f_{S}}{1-f_{S}}=\frac{d x_{i}^{L}}{x_{i}^{L}} .
$$

Assuming a constant $K_{i}$, we obtain eq. (5) after a simple integration of the above equation from 0 to $f_{S}$ on the left side and $x_{i}^{0}$ to $x_{i}^{L}$ on the right side.

\subsubsection{Partial equilibrium model}

The numerical algorithm for the partial equilibrium model can be obtained by simply modifying the scheme for the Scheil-Gulliver model presented in the preceding section. At each iteration step, the composition and amount of the remaining liquid will be adjusted according to the partial equilibrium assumption. For simplicity, carbon will be the only interstitial element to be discussed in the present work. Accepting that there are complete interstitial and negligible subsitutional solute back diffusions in the solid, we shall allow carbon to move freely among the liquid and all portions of the solid phases formed at the present and previous temperature steps, so that its chemical potential $\mu_{C}$ becomes equal everywhere throughout the whole system. At the same time, substitutional solutes shall be completely "frozen" in the remaining liquid and each portion of the solid formed, which means that their $u$-fractions (defined as $\left.u_{i \neq C}=x_{i \neq C} /\left(1-x_{C}\right)\right)^{14)}$ shall not change during the back diffusion of carbon. Therefore, at any step $k$, the following relation can thus be applied to each portion of the solid phases formed

$$
\left.{ }^{p} \mu_{C}^{S_{j}}\right|_{p_{u_{i \neq C}} S_{j}}=\left.{ }^{k} \mu_{C}^{L}\right|_{{ }^{u_{i \neq C}^{L}}}
$$

where $p$ denotes the portion formed at the step $p(1 \leq p \leq k)$. eq. (11) yields $j \times k$ independent relations if $j$ solid phases have formed in all the steps. We need one more equation to obtain $1+j \times k$ unknown variables, i.e., the new content of carbon in the remaining liquid and the portions of solid phases, $\left({ }^{k} x_{C}^{L}\right)^{\prime}$ and $\left({ }^{p} x_{C}^{S_{j}}\right)^{\prime}$. By considering the mass conservation of the interstitial element, we get the necessary 


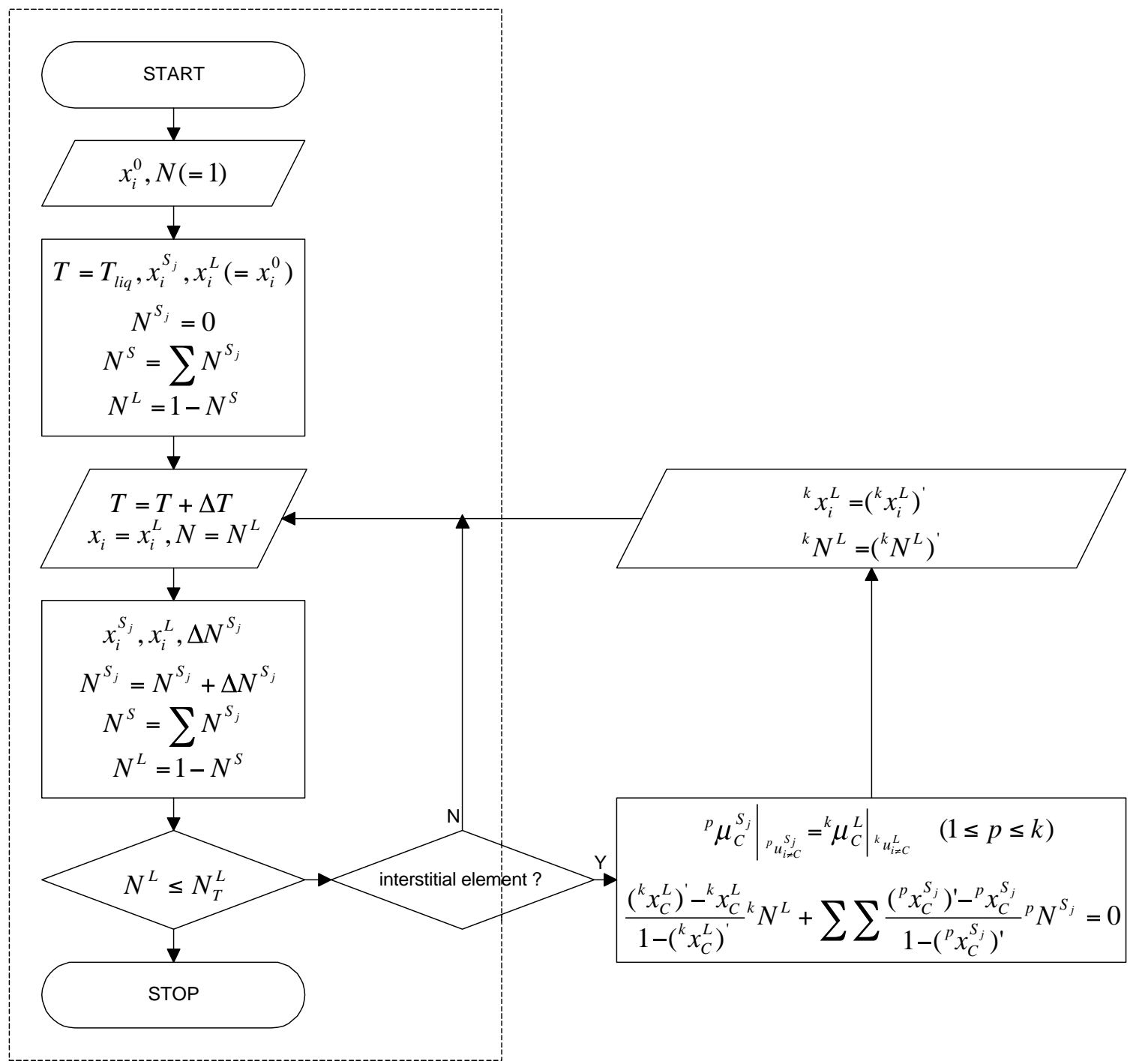

Fig. 1 Computational scheme for partial equilibrium solidification.

equation

$$
\frac{\left({ }^{k} x_{C}^{L}\right)^{\prime}-{ }^{k} x_{C}^{L}}{1-\left({ }^{k} x_{C}^{L}\right)^{\prime}}{ }^{k} N^{L}+\sum \sum \frac{\left({ }^{p} x_{C}^{S_{j}}\right)^{\prime}-{ }^{p} x_{C}^{S_{j}}}{1-\left({ }^{p} x_{C}^{S_{j}}\right)^{\prime}}{ }^{p} N^{S_{j}}=0
$$

where ${ }^{k} N^{L}$ and ${ }^{p} N^{S_{j}}$ are the amount of the remaining liquid and the $p$-th portion of solid phase $S_{j}$ before the carbon back diffusion at the step $k$. Having obtained $\left({ }^{k} x_{C}^{L}\right)^{\prime}$ and $\left({ }^{p} x_{C}^{S_{j}}\right)^{\prime}$, we may easily get the new content of substitutional solutes in the liquid and solid phase(s), $\left({ }^{k} x_{i \neq C}^{L}\right)^{\prime}$ and $\left({ }^{p} x_{i \neq C}^{S_{j}}\right)^{\prime}$, as well as the new amount or fraction of the liquid and solid(s), $\left({ }^{k} N^{L}\right)^{\prime}$ and $\left({ }^{p} N^{S_{j}}\right)^{\prime}$, using the relations:

$$
\begin{aligned}
\left({ }^{k} x_{i \neq C}^{L}\right)^{\prime} & =\frac{1-\left({ }^{k} x_{C}^{L}\right)^{\prime}}{1-{ }^{k} x_{C}^{L}}{ }^{k} x_{i \neq C}^{L} \\
\left({ }^{p} x_{i \neq C}^{S_{j}}\right)^{\prime} & =\frac{1-\left({ }^{p} x_{C}^{S_{j}}\right)^{\prime}}{1-{ }^{p} x_{C}^{S_{j}}}{ }^{{ }^{S}} x_{i \neq C}^{S_{j}} \\
\left({ }^{k} N^{L}\right)^{\prime} & =\frac{1-{ }^{k} x_{C}^{L}}{1-\left({ }^{k} x_{C}^{L}\right)^{\prime}}{ }^{k} N^{L}
\end{aligned}
$$

$$
\left({ }^{p} N^{S_{j}}\right)^{\prime}=\frac{1-{ }^{p} x_{C}^{S_{j}}}{1-\left({ }^{p} x_{C}^{S_{j}}\right)^{\prime}}{ }^{p} N^{S_{j}}
$$

After this partial equilibrium adjustment to the phase composition and amount, the remaining liquid will be in a metastable equilibrium state and some solid phase(s) would form if an equilibrium calculation is performed on it at the current temperature step. However, we shall not perform such an equilibrium calculation, which will apparently lead to smaller and smaller adjustment until the new liquid composition is on the liquidus surface at the current temperature. We will use the metastable liquid directly at the next temperature step for the local equilibrium calculation and subsequent partial equilibrium manipulation. The full computational procedure is shown in Fig. 1.

Now let's look back at the partial equilibrium condition eq. (11). As we said before, it consists of $j \times k$ independent relations if $j$ solid phases have formed in all $k$ steps. For commercial alloys, a normal simulation has hundreds or over one thousand temperature steps, depending on the freezing range and temperature step. As the simulation continues, $k$ becomes larger and larger and $j$ also increases, there will be a formidable number of equations to solve. In order to sim- 
plify the problem we shall approximate the interstitial back diffusion by summing up the amount and calculating an average composition for each kind of solid phase thus formed, and then even out the interstitial chemical potential among the remaining liquid and each solid phase of the average composition. As a consequence, at any step $k$, eqs. (11) and (12) become

$$
\begin{aligned}
& \left.{ }^{k} \bar{\mu}_{C}^{S_{j}}\right|_{{ }^{\prime} \bar{u}_{i \neq C} S_{j}}=\left.{ }^{k} \mu_{C}^{L}\right|_{k_{u_{i \neq C}^{L}}} \\
& \frac{\left({ }^{k} x_{C}^{L}\right)^{\prime}-{ }^{k} x_{C}^{L}{ }^{k}}{1-\left({ }^{k} x_{C}^{L}\right)^{\prime}} N^{L}+\sum \frac{\left({ }^{k} \bar{x}_{C} S_{j}\right)^{\prime}-{ }^{k} \bar{x}_{C} S_{j}}{1-\left({ }^{k} \bar{x}^{S}\right)^{\prime}} N^{S_{j}}=0
\end{aligned}
$$

where ${ }^{k} \bar{\mu}_{C}^{S_{j}}$ is the average chemical potential of carbon in the solid phase $S_{j}$ of an average $u$-fraction of substitutional solute ${ }^{k} \bar{u}_{i \neq C} S_{j} \cdot{ }^{k} \bar{x}_{C} S_{j}$ and $\left({ }^{k} \bar{x}_{C} S_{j}\right)^{\prime}$ are the average mole fractions of carbon in the solid phase $S_{j}$ before and after evening out the chemical potential. In this simplified approach, we are not tracking the properties of individual portion solidified at each temperature step but the average ones for each kind of solid phase thus formed. Therefore, instead of solving a frightening $1+j \times k$ dimensional problem, we are now comfortably dealing with a $1+j$ one and will have a tremendous gain in computational time. However, admittedly, due to this simplification we will lose the ability to follow the change of composition in each individually solidified part and cannot obtain the actual solute distribution at the terminating step $k_{T}$, i.e., ${ }^{p} x_{i}^{S_{j}}\left(1 \leq p \leq k_{T}\right)$. Nevertheless, we may still use the solid composition at the solid/liquid interface at each temperature step to represent the final solute distribution for substitutional elements, and, fortunately, this may give even better agreements between the calculation and measurements than the original approach which solves the problem rigorously by employing eqs. (11) to (16). In fact, the unavoidable back diffusion of substitutional elements in a real alloy, even though very small, will perhaps be large enough or more than enough to cancel the composition change of substitutional solute due to the interstitial back diffusion.

\section{Results and Discussion}

A computing tool, called SCHEIC (SCHEil with Interstitial Carbon), has been developed for simulating partial equilibrium solidification by using the numerical method presented in the preceding section. The primary goal of devising this tool is to take into account of back diffusion of carbon in the solidification of steels, but the usage is not restricted to carbon and steels. Alloys with other interstitial element(s) can also be treated. With the aid of the TQI (Thermodynamic calCUlation Interface) ${ }^{20)}$ an application program interface of the Thermo-Calc, we have made it possible for this computational tool to use the thermodynamic data directly from databases available to the Thermo-Calc software package. With use of this new tool, it will be as easy to perform a partial equilibrium simulation as to do a Scheil-Gulliver calculation inside the Thermo-Calc system, but the results will be closer to reality if interstitial elements are involved. A few examples will now be demonstrated.

Ono et ll. $^{21)}$ has recently reported their experimental re- sults on $\mathrm{Fe}-10.84 \% \mathrm{Cr}-0.95 \% \mathrm{C}$ and $\mathrm{Fe}-5.34 \% \mathrm{Cr}-0.92 \% \mathrm{C}$ alloys solidified at a cooling rate of $0.167 \mathrm{~K} / \mathrm{s}$. Using SCHEIC and Thermo-Calc together with the $\mathrm{TCFE}^{22)}$ database, partial equilibrium and Scheil-Gulliver simulations were performed for these two steels. In addition, DICTRA, a software with full integration of thermodynamics and kinetics, has also been employed to corroborate the measurements and our simple calculation results. The $\mathrm{TCFE}^{22)}$ thermodynamic database, MOB2 ${ }^{23)}$ mobility database, and experimental parameters, i.e., cooling rate and secondary arm spacing, were used in DICTRA simulations. It should be mentioned that in this study, all partial equilibrium and Scheil-Gulliver simulations have been terminated when the fraction of the remaining liquid become less than $1 \%$.

Figure 2 shows the agreement between the calculated and experimental results on the microsegregation of $\mathrm{Cr}$ in these two steels. In Fig. 2(a), it can be seen that the DICTRA simu-
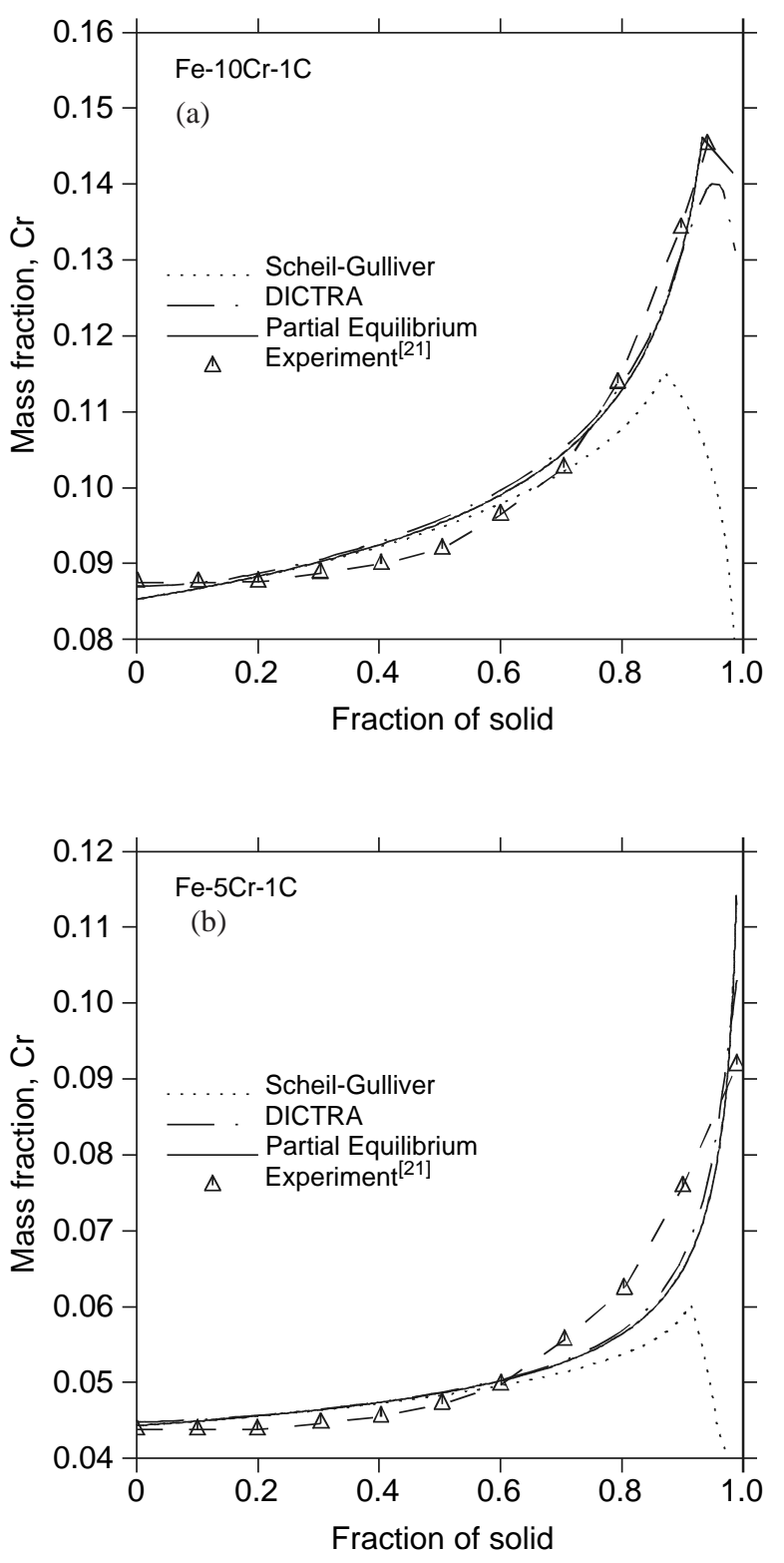

Fig. 2 Microsegregation of $\mathrm{Cr}$ in $\mathrm{Fcc}$ phase in steel (a) $\mathrm{Fe}-10.84 \% \mathrm{Cr}$ $0.95 \% \mathrm{C}$ and (b) $\mathrm{Fe}-5.34 \% \mathrm{Cr}-0.92 \% \mathrm{C}$. The dashed lines serve as a guideline for the experimental data. ${ }^{21)}$ 


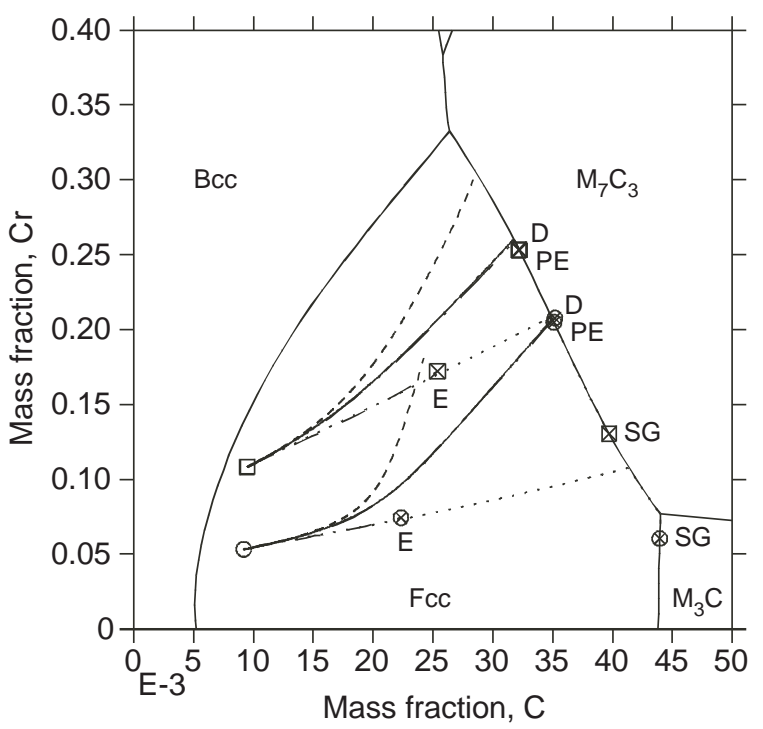

Fig. 3 Liquidus surface projection and solidification paths of $\mathrm{Fe}-10.84 \% \mathrm{Cr}-0.95 \% \mathrm{C}$ and $\mathrm{Fe}-5.34 \% \mathrm{Cr}-0.92 \% \mathrm{C}$ steel. Square and circle indicate the start for steel $\mathrm{Fe}-10.84 \% \mathrm{Cr}-0.95 \% \mathrm{C}$ and $\mathrm{Fe}-5.34 \% \mathrm{Cr}-$ $0.92 \% \mathrm{C}$, respectively. Symbols with $\times$ mark the end points according to various simulations: E-Equilibrium, SG-Scheil-Gulliver, D-DICTRA, PE-Partial Equilibrium. The styles of lines follow those in Fig. 2 except that the dashed lines represent the results from the analytical method.

lation result at $f_{S}=0$ is almost exactly the same as the measurement, and the partial equilibrium value is slightly lower. This difference is due to the fact that the back diffusion of $\mathrm{Cr}$ has been completely neglected in the partial equilibrium treatment. Overall, the two simulation results agree with each other better than with the experimental results, which means that the partial equilibrium assumption and numerical algorithm are reasonable and satisfactory, but the thermodynamic database used may need improvement or some of the experimental measurements may involve a large uncertainty.

By comparing with the Scheil-Gulliver simulation which neglects back diffusion completely, we can clearly see from Fig. 2 that the microsegregation of $\mathrm{Cr}$ in the primary Fcc phase is enhanced due to the back diffusion of carbon, a fact observed by many investigators but never fully explained. Considering steel $\mathrm{Fe}-5.34 \% \mathrm{Cr}-0.92 \% \mathrm{C}$ where the effect is most prominent, it is apparent that this is largely because the formation of eutectic mixture is postponed (see Fig. 2(b)) and the enrichment of chromium in the liquid becomes more and more severe towards the end of solidification. The change of the liquid composition in the solidification process is shown in Fig. 3. From this plot, we see that the solidification path is greatly changed due to the back diffusion of carbon. As a matter of fact, it is the change of solidification path that causes the delay of eutectic formation. Because of the change of solidification path, the variation of partition coefficient of $\mathrm{Cr}$ for the partial equilibrium solidification is also different from that for the Scheil-Gulliver simulation, see Fig. 4. This can explain why the microsegregation curve for partial equilibrium has a different curvature from that for Scheil-Gulliver simulations in Fig. 2, where we can see that the former one lies more and more above the latter one as the solidification continues. From Fig. 2, we can also see that there is a sharp decrease in $\mathrm{Cr}$ content of the eutectic Fcc phase towards the

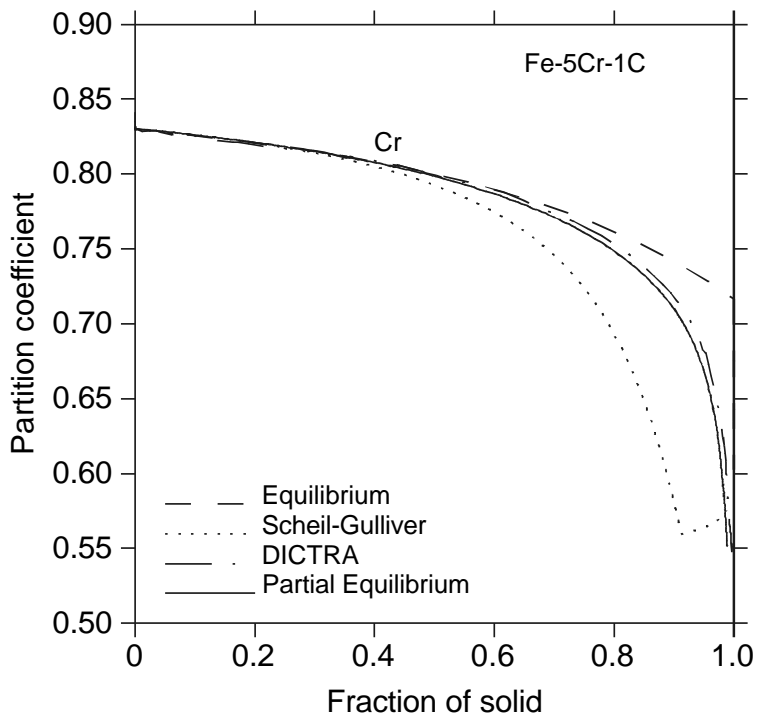

Fig. 4 Partition coefficient of $\mathrm{Cr}$ varying with the fraction of solid in steel $\mathrm{Fe}-5.34 \%-0.92 \% \mathrm{C}$.

end of solidification. This is caused by the formation of $\mathrm{Cr}$ carbide in the eutectic reaction.

In Fig. 3, the results from a direct combination of eq. (4) for $\mathrm{C}$ and eq. (5) for $\mathrm{Cr}$ by using thermodynamically calculated partial coefficients are also shown. It is clear that these results deviate severely from those of the real partial equilibrium calculation and DICTRA simulation. Therefore, one must be extremely careful when being tempted to use this naive "partial equilibrium" approach.

The solid fraction vs temperature curves for the $\mathrm{Fe}-$ $10.84 \% \mathrm{Cr}-0.95 \% \mathrm{C}$ and $\mathrm{Fe}-5.34 \% \mathrm{Cr}-0.92 \% \mathrm{C}$ steels are depicted in Fig. 5. The agreement between the simple partial equilibrium simulation and the more sophisticated DICTRA simulation is apparent. A favorable comparison with the experimental results on the eutectic fractions and freezing ranges is given in Table 1.

The solidification of the $\mathrm{Fe}-10.84 \% \mathrm{Cr}-0.95 \% \mathrm{C}$ and $\mathrm{Fe}-$ $5.34 \% \mathrm{Cr}-0.92 \% \mathrm{C}$ alloys involves only two solid phases, and can be modeled by using DICTRA conveniently although it needs more computational time. For multi-solid cases in conventional commercial steels, DICTRA is not applicable, but SCHEIC can be used with the same ease. Figure 6 shows the predicted solid fraction vs temperature curves for the AISI M2 steel. ${ }^{24)}$ The solute compositions used in the calculations are $0.88 \% \mathrm{C}, 0.30 \% \mathrm{Si}, 0.32 \% \mathrm{Mn}, 3.9 \% \mathrm{Cr}, 0.36 \% \mathrm{Ni}, 4.9 \% \mathrm{Mo}$, $0.1 \% \mathrm{Cu}, 0.3 \% \mathrm{Co}, 6.1 \% \mathrm{~W}$, and $1.9 \% \mathrm{~V}$. Although the formation of $\mathrm{M}_{7} \mathrm{C}_{3}$ instead of $\mathrm{M}_{2} \mathrm{C}$ is not in conform with the experimental information, ${ }^{24)}$ and indicates a need to improve the thermodynamic database, it is remarkable that the estimated freezing range from the partial equilibrium calculation is very close to the experimental ones which was measured at an average cooling rate of 2.0 and $0.5 \mathrm{~K} / \mathrm{s}^{24)}$

From the above examples, it may be concluded that the partial equilibrium approach is a big improvement to the ScheilGulliver method, and the results obtained from the partial equilibrium approach is very close to that from DICTRA in the solidification of the steels investigated. This success suggests that the basic partial equilibrium assumption, i.e., com- 
Table 1 Calculated and experimental results on the eutectic fraction and freezing range of solidified $\mathrm{Fe}-10.84 \% \mathrm{Cr}-0.95 \% \mathrm{C}$ and $\mathrm{Fe}-5.34 \% \mathrm{Cr}-0.92 \% \mathrm{C}$ steels.

\begin{tabular}{lccc} 
& \multicolumn{2}{c}{$\mathrm{Fe}-10.84 \% \mathrm{Cr}-0.95 \% \mathrm{C}$} & $\mathrm{Fe}-5.34 \% \mathrm{Cr}-0.92 \% \mathrm{C}$ \\
\cline { 2 - 4 } & \multicolumn{1}{c}{ Eutectic fraction } & Freezing range, K & Eutectic fraction \\
\hline Lever-rule & 0 & 103 & 0 \\
Scheil-Gulliver & $11.69 \%$ & 211 & $7.65 \%$ \\
DICTRA & $5.50 \%$ & 159 & $0.43 \%$ \\
Partial equil. & $6.74 \%$ & 159 & $1.14 \%$ \\
Experiment $^{21)}$ & $5.54 \%$ & 187 & 187 \\
\hline
\end{tabular}
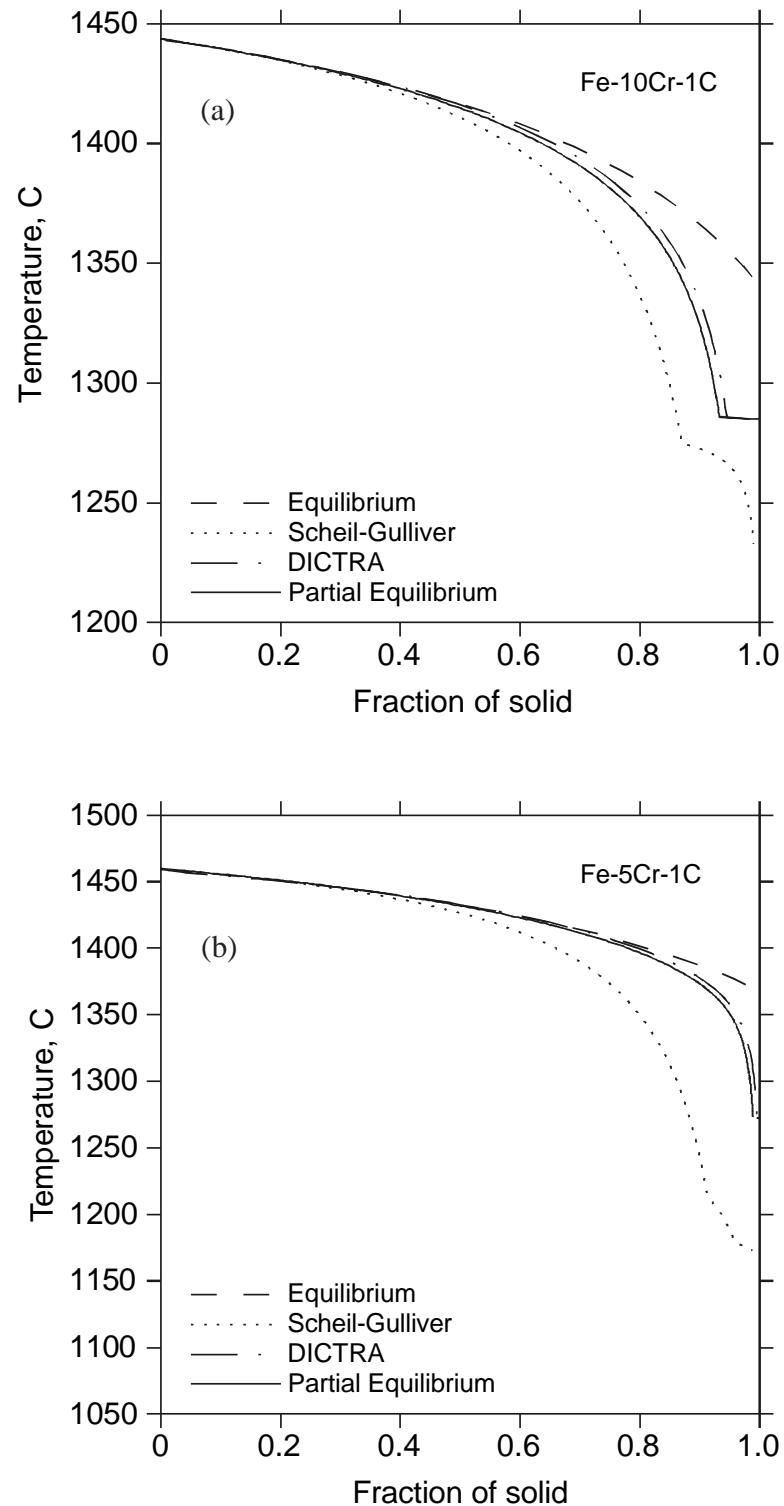

Fig. 5 Solid fraction vs temperature curve for steels (a) $\mathrm{Fe}-10.84 \% \mathrm{Cr}-$ $0.95 \% \mathrm{C}$ and (b) $\mathrm{Fe}-5.34 \% \mathrm{Cr}-0.92 \% \mathrm{C}$.

plete interstitial and negligible substitutional back diffusion, is reasonable for the case studied. As a matter of fact, we can verify this point by making a rough estimation of the ClyneKurz back-diffusion parameter using eqs. (2) and (3) although the analytic method is not thermodynamically consistent all the time. For the austenitic primary phase, the diffusion coefficient of carbon is in the order of $10^{-9} \mathrm{~m}^{2} \mathrm{~s}^{-1}$ and that of

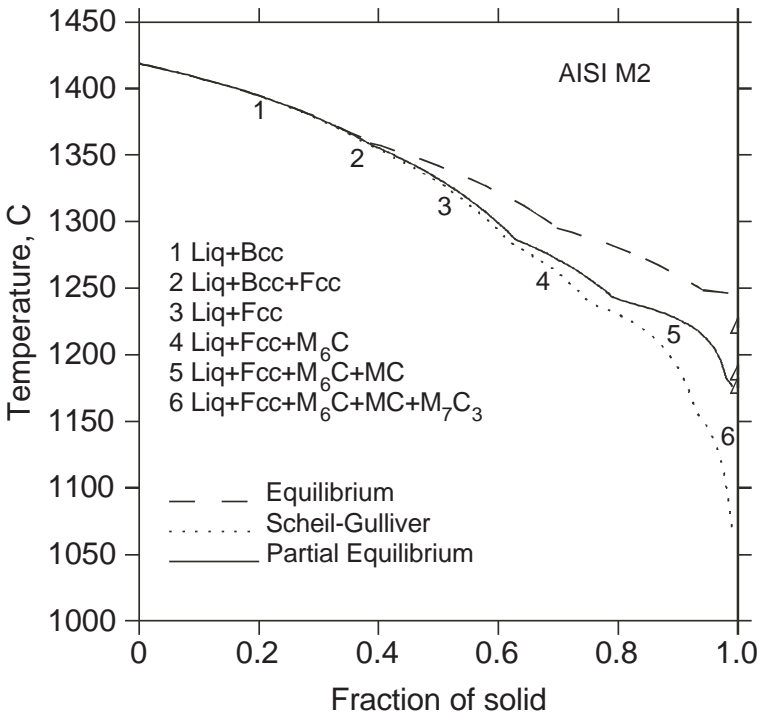

Fig. 6 Predicted solid fraction vs temperature curves of AISI M2 steel. The triangles mark the experimental solidus temperatures for the cooling rates of $0.1,0.5$, and $2.0 \mathrm{~K} / \mathrm{s}$, respectively. ${ }^{24}$ )

substitutional elements of $10^{-13} \mathrm{~m}^{2} \mathrm{~s}^{-1} \cdot{ }^{23)}$ Knowing that the secondary dendrite arm spacing and local freezing time are in the order of $10^{-4} \mathrm{~m}$ and $10^{3} \mathrm{~s}$, respectively, we have $\alpha=400$ for carbon and $\alpha=0.04$ for substitutional elements. Examining the plot of the Clyne-Kurz function given in Fig. 7, we see that $\Omega(\alpha)$ is very close to the higher limiting value 0.5 for carbon and close to 0 for subsitutional elements, which would then lead to eqs. (4) and (5), respectively, according to the Clyne and Kurz equation. This indicates that the back diffusion of carbon is almost complete and that of substitutional elements is negligible.

Now let us make a similar estimation for the ferritic primary phase. Using the same values for the austenite except the diffusion coefficient of substitutional elements, which is about $10^{-11} \mathrm{~m}^{2} \mathrm{~s}^{-1}$ for the ferrite, ${ }^{23)}$ we have $\alpha=400$ for carbon and $\alpha=4$ for substitutional elements. In this case, we know from Fig. 7 that the back diffusion of substitutional elements is no longer negligible, but rather close to complete. Therefore, in steels dominated by the ferritic primary phase, the partial equilibrium approach is not expected to give very good predictions although it certainly gives better results than the Scheil-Gulliver method. Maybe it could be improved by treating $\mathrm{Cr}$ as a fast-diffusing element in ferrite. However, if all alloying elements are fast-diffusing in ferrite, then the model would approach the lever-rule model.

Simulations have been carried out for several ferritic steels 


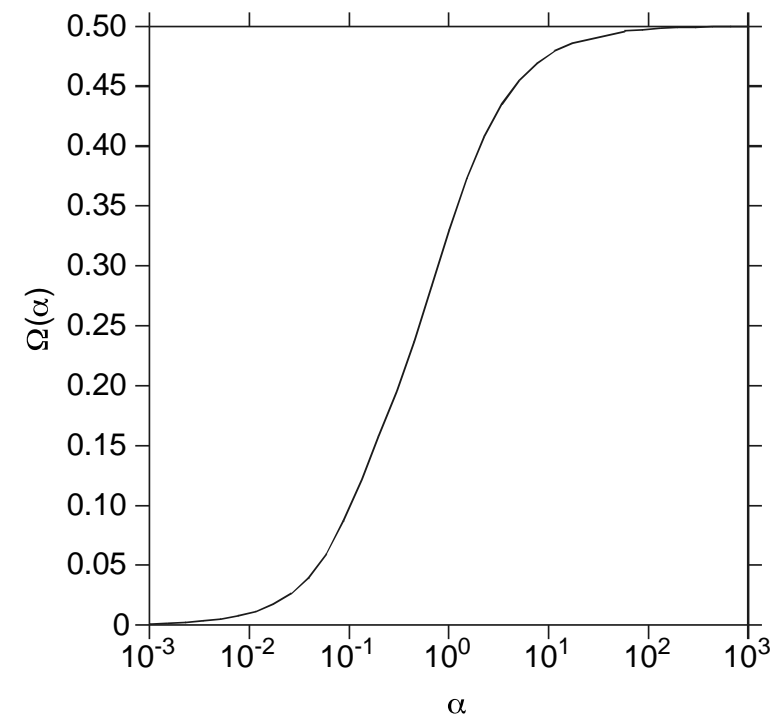

Fig. 7 Variation curve of the Clyne-Kurz function $\Omega(\alpha)$ with $\alpha$. The up end means that the back diffusion is complete; the low end means that the back diffusion is negligble.

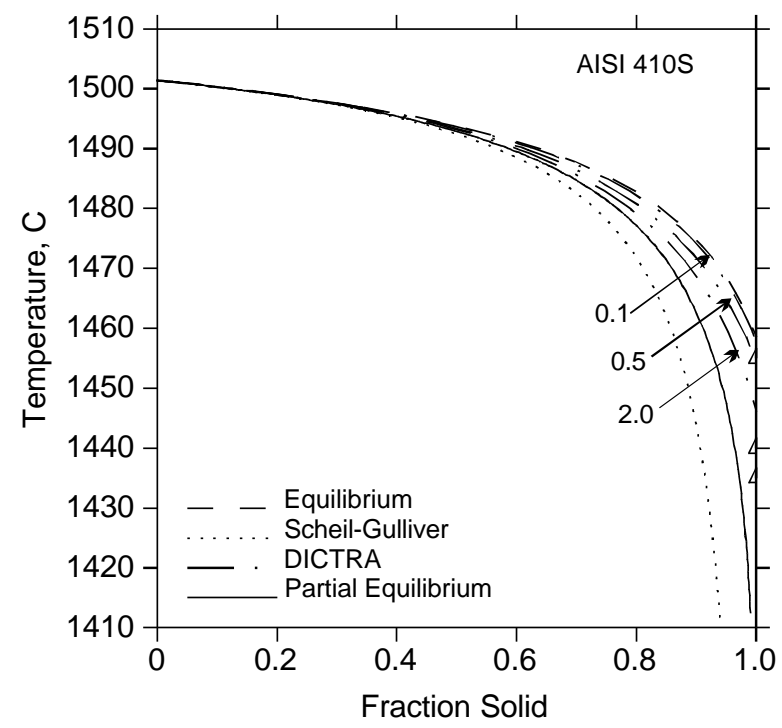

Fig. 8 Predicted solid fraction vs temperature curve for AISI 410S steel. Three DICTRA results have been marked with the corresponding cooling rate in $\mathrm{K} / \mathrm{s}$. The triangles mark the experimental solidus temperatures for the cooling rates of $0.1,0.5$, and $2.0 \mathrm{~K} / \mathrm{s}$, respectively. ${ }^{24)}$

experimentally investigated in Ref. 24). It was found that for these steels the solidification behavior is indeed closer to the lever-rule than the partial equilibrium model. Here we take AISI $410 S^{24)}$ as an example. The results on the solid fraction vs temperature relation are depicted in Fig. 8. For DICTRA calculations, three experimental cooling rates ${ }^{24}$ have been used. From the plot, it can be seen that even under the largest cooling rate, the DICTRA curve is closer to that of the leverrule than the partial equilibrium, although the experimental solidus points are slightly lower than the DICTRA predictions. This is more or less true for the microsegregation ratio if the equilibrium chromium concentration in ferrite at each temperature step is drawn for equilibrium solidification, see Fig. 9, although we know that there should be no microsegregation in the lever-rule model.

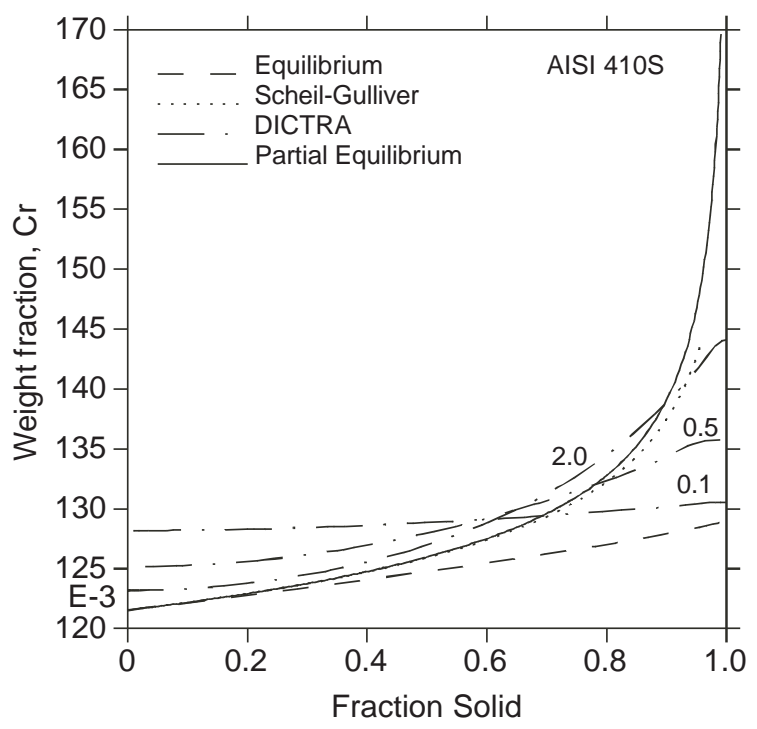

Fig. 9 Predicted microsegregation of $\mathrm{Cr}$ in AISI 410S steel. DICTRA results have been marked with the corresponding cooling rate in K/s. The equilibrium curve shows the change of equilibrium composition in $\mathrm{Bcc}$ phase during the equilibrium solidification process.

\section{Summary}

The solidification model called partial equilibrium simulation is a reasonable approximation for steels except for those dominated by the ferrite as the primary phase. A simple numerical scheme has been described for the application of the partial equilibrium solidification in multi-component and multi-phase systems. Based on this scheme, a general computing tool capable of using Thermo-Calc databases directly has been developed. Agreement between calculated and experimental as well as DICTRA results have been obtained on the microsegregation, fraction of eutectic, and freezing range of several steels. This confirms that the partial equilibrium approximation is reasonable for the steels investigated and suggests that the proposed numerical scheme is satisfactory. However, for steels where primary precipitation of ferrite predominates, the lever-rule may give better results. According to our simulation, the influence of carbon on the microsegregation of chromium in the primary Fcc phase in the $\mathrm{Fe}-\mathrm{Cr}-\mathrm{C}$ system is due to the change of solidification path upon the back diffusion of carbon.

\section{Acknowledgements}

Part of this work is financially supported by the EC-funded VESPISM project. The authors wish to thank Prof. M. Hillert for constructive suggestions.

\section{REFERENCES}

1) A. Borgenstam, A. Engström, L. Höglund, and J. Ågren: J. Phase Equilibria 21 (2000) 269-280.

2) G. M. Gulliver: J. Inst. Metals 9 (1913) 120-157.

3) E. Scheil: Z. Metallkd., 34 (1942) 70-72.

4) M. C. Flemings: Solidification Processing, (McGraw-Hill, New York, NY, 1974).

5) N. Saunders and A. P. Miodownik: CALPHAD Calculation of Phase Diagrams: A Comprehensive Guide, (Pergamon, New York, NY, 1998).

6) M. Hillert, L. Höglund and M. Schalin: Metall. Mater. Trans. A 30A 
(1999) 1635-1641.

7) H. Fredriksson and L. Hellner: Scand. J. Metall. 3 (1974) 61-68.

8) E. Kozeschnik: Metall. Mater. Trans. A 31A (2000) 1682-1684.

9) H. D. Brody and M. C. Flemings: Trans. TMS-AIME 236 (1966) 615624.

10) T. W. Clyne and W. Kurz: Metall. Trans. A 12A (1981) 965-971.

11) I. Ohnaka: Trans. ISIJ 26 (1986) 1045-1051.

12) S. Kobayashi: Trans. ISIJ 28 (1988) 728-735.

13) L. Nastac and D. M. Stefanescu: Metall. Trans. A 24A (1993) 21072118

14) M. Hillert: Phase Equilibrium, Phase Diagrams and Phase Transformations, (Cambridge University Press, New York, NY, 1998).

15) L. Kaufman and H. Bernstein: Computer Calculation of Phase Diagrams, (Academic Press, New York, NY, 1970).

16) CALPHAD-Computer Coupling of Phase Diagrams and Thermochemistry, (Pergamon, New York, NY).
17) B. Sundman, B. Jansson and J.-O. Andersson: CALPHAD 9 (1985) 153-190.

18) B. Sundman and I. Ansara: in The SGTE Casebook-Thermodynamics at Work, ed. K. Hack (The Institute of Materials, London, UK, 1996), pp. 94-98.

19) Q. Chen and B. Sundman: unpublished work, 1996.

20) B. Sundman and Q. Chen, "Thermodynamic Calculation Interface (TQ): Programmers' Guide and Examples", (Thermo-Calc AB, Stockholm, 2001).

21) Y. Ono, T. Takechi and K. Ogi: J. Japan Inst. Metals 57 (1993) 432-439.

22) TCFE-Thermo-Calc AB steel database, Thermo-Calc AB, Stockholm, 1998.

23) MOB2-Mobility database, Thermo-Calc AB, Stockholm, 1998.

24) "A Guide to the Solidification of Steels", Jernkontoret, Stockholm, 1977. 